tissues, but at tenfold lower levels than in prostate. The only prostate cancer cell line that expresses $1 \mathrm{~B} 10$ is an androgen-sensitive cell line, LNCaP, suggesting that the gene might be androgen-regulated. Cloning of the full-length cDNA has so far resulted in $4.6 \mathrm{~kb}$ of the transcript. The $3^{\prime}$ end of the coding region is significantly homologous to a prostate-specific transmembrane protein, STEAP, but the rest of the sequence does not show homology to any known gene. Cloning of the $5^{\prime}$ end of the cDNA and functional studies are now in progress.

Porter, Peggy L.

\section{Allelic imbalance in routinely processed breast tumors determined by affymetrix HuSNP array}

Elizabeth Schubert, Laura Cousens, Li Hsu, Jeri Glogovac, Brian Reid, Steve Self, Peter Rabinovich \& Peggy Porter

Fred Hutchinson Cancer Research Center, Seattle, Washington, USA

Analysis of allelic loss in archival tumor specimens using array technology is constrained by the quality and quantity of available tissue. A prototype Affymetrix HuSNP array was shown to provide reliable and reproducible assessment of allelic imbalance for 440 SNPs in frozen esophageal tumors (Mei-2000). However, the commercially-available Affymetrix HuSNP array $(1,494 \mathrm{SNPs})$ has not been validated for the assessment of allelic imbalance in tumors processed by standard pathology methods. We tested the HuSNP assay in duplicate on breast specimens using both formalin-fixed and frozen, tumor and normal tissue taken from a single patient ( 16 arrays). Tumor cells were purified using bivariate cytokeratin/DNA flow sorting; normal breast served as constitutive normal. STR typing on 3 chromosomes validated regions of allelic imbalance. Allele calls from the HuSNP array averaged $95 \%$ reproducibility between duplicates and $94 \%$ concordance between the fixed and frozen samples. We also tested DNA from the same samples that was subjected to whole genome amplification (Primer Extension Pre-amplification, PEP) prior to array analysis. Although overall signal intensities were lower, the data from the PEPed material was reproducible in duplicates and concordant between sample types at similar rates to genomic DNA. Results from genomic normal tissue DNA averaged informative $(\mathrm{AB})$ calls at 379 loci over all chromosomes. Although data points were clustered and large segments of chromosomes were not informative by this technique, our data indicated that the Affymetrix HuSNP assay could potentially provide a low resolution genome-wide analysis of allelic imbalance in routinely processed pathology specimens.

Randazzo, Filippo

\section{Cancer pathway analysis, DNA microarrays, and the identification of therapeutic targets}

Filippo Randazzo ${ }^{1}$, Pablo Garcia ${ }^{1}$, Joel Berger ${ }^{1}$, Anna Kelly ${ }^{1}$, John Fuller ${ }^{1}$, Pauline Lee ${ }^{1}$, James Stephans ${ }^{1}$, Mark Boyle ${ }^{1}$, Eddie Moler $^{1}$, Eric Dahlstrom ${ }^{1}$, Mala Sinha ${ }^{1}$,

Christoph Reinhard ${ }^{1}$, Robert Warren ${ }^{2}$, Emily Bergsland ${ }^{2}$, Terry Brentnall ${ }^{3}$, Mary Bronner ${ }^{3}$, Mark van der Laan ${ }^{4}$, Katie Pollard ${ }^{4}$, Rusty Williams ${ }^{1} \&$ Jaime Escobedo ${ }^{1}$

${ }^{1}$ Chiron Corporation, Emeryville, California, USA

${ }^{2}$ University of California San Francisco, San Francisco, California, USA

${ }^{3}$ University of Washington, Seattle, Washington, USA

${ }^{4}$ University of California Berkeley, Berkeley, California, USA

We are discovering new drug targets for cancer using a combination of genomic and functional assay technologies. As part of our discovery and validation process we use DNA microarrays to determine the patterns of gene expression in normal tissue, primary tumors and metastatic tumors across a cancer patient population. Tens of thousands of genes, including cancer genes discovered by highly sensitive gene profiling technology, are placed on our DNA microchips. Our DNA microarray technology is highly sensitive and reproducible, allowing us to assay laser capture microdissected material from human biopsy samples. We analyze differential gene expression patterns in the cancer patient population using new clustering approaches. These patterns are, in turn, analyzed against our database of gene expression profiles obtained from cell lines in which pathways have been manipulated, and pathways that are altered in patients are identified. We then further evaluate selected differentially expressed genes, along with their pathways, as potential targets for diagnostic and therapeutic approaches using functional analysis.

Ratner, Nancy

[12]

\section{Gene expression analysis of NF1 mutant Schwann cells}

\author{
Nancy Ratner ${ }^{1}$, Shyra Miller ${ }^{1} \&$ Jeffrey DeClue ${ }^{2}$
}

${ }^{1}$ University of Cincinnati College of Medicine, Cincinnati, Ohio, USA

${ }^{2}$ National Cancer Institute, National Institutes of Health, Bethesda, Maryland, USA

Schwann cells constitute the major cell population in neurofibromas, tumors that disfigure patients with the common inherited disease neurofibromatosis type 1. It is not known how abnormalities in neurofibroma Schwann cells contribute to tumor formation. We developed phenotypic assays that distinguish normal Schwann cells from Schwann cells (mouse and human) that lack the NF1 tumor suppressor protein, a GTPase-activating protein for Ras proteins. To define genes that may underlie these phenotypic changes we hybridized cDNA from wild-type and Nf1 mutant mouse Schwann cells to microarrays from Incyte Genomics and compared gene expression levels. We carried out four arrays, each in duplicate, comparing cells of various phenotypes $\left(\mathrm{Nfl}^{+/-}, \mathrm{Nf}^{--}, \mathrm{Nfl}^{-/-}\right.$TXF [a proliferative Schwann cell population] and $\mathrm{Nf1}^{-/-} \mathrm{TXF}$ treated with a farnesyl transferase inhibitor [a drug class in clinical trials in NF1] to wild-type Schwann cells. Thirteen changes of greater than fourfold were identified in $\mathrm{Nf}^{-/-}$Schwann cells. Larger numbers of genes were differentially expressed in $\mathrm{Nf}^{-l-}$ TXF Schwann cells; some were sensitive to farnesyl transferase inhibitor. We report on the reproducibility of the data and confirm the RNA and protein levels for some identified genes. We have also begun an effort to share array data among laboratories studying neurofibromatosis as part of an international consortium. By applying these methods to NF1 model systems we hope to learn how neurofibromin serves as a tumor suppressor and to identify potential biomarkers and therapeutic targets for improved management of neurofibromatosis. 